\title{
Hsa_circ_0008537 facilitates liver carcinogenesis by upregulating MCL1 and Snail1 expression via miR-153-3p
}

\author{
GE YANG, XIANYONG LI, JINGBO LIU, SHENGJIE HUANG, YAGUANG WENG, \\ JING ZHU, DAIQIONG LIN and OU JIANG
}

\begin{abstract}
Department of Clinical Laboratory, Affiliated Neijiang Second People's Hospital of Southwest Medical University, Neijiang, Sichuan 641000, P.R. China
\end{abstract}

Received May 13,2020; Accepted November 3, 2020

DOI: $10.3892 / o r .2021 .7941$

\begin{abstract}
The biological functions of circular RNAs in liver tumorigenesis have been well demonstrated by a number of studies. Nevertheless, to the best of our knowledge, the role and mechanism of action of hsa_circ_0008537 (circ_0008537) in liver cancer pathogenesis remain undetermined. In the present study, circ_0008537 expression was associated with the GLI3 gene and was markedly increased in liver cancer tissue specimens and cells. High expression levels of circ_0008537 exhibited a poor prognosis. In addition, circ_0008537 overexpression resulted in an increased proliferation, migration and invasion of liver cancer cells, whereas circ_0008537 knockdown exhibited opposite effects. circ_0008537 acted as a sponge of microRNA-153-3p (miR-153-3p), and a negative correlation was observed between circ_0008537 and miR-153-3p expression in liver cancer. Transfection with miR-153-3p further abolished the effects of circ_0008537 on the malignant behavior of liver cancer cells. Furthermore, circ_0008537 indirectly affected the expression levels of pro-survival protein myeloid cell leukemia 1 (MCL1) and snail family zinc finger 1 (Snail1) via miR-153-3p in liver cancer cells. In conclusion, the data indicated that circ_0008537 facilitated liver carcinogenesis by indirectly regulating miR-153-3p and leading to the release of MCL1 and Snaill.
\end{abstract}

\section{Introduction}

Primary liver cancer ranks as the sixth most frequently diagnosed cancer and the second most common cause of tumor-related deaths in the world (1). Due to the poor prognosis of this disease, its mortality has increased, reaching 30,160 deaths in 2020 (2). As the most common subtype of primary

Correspondence to: $\mathrm{Dr} \mathrm{Ou}$ Jiang, Department of Clinical Laboratory, Affiliated Neijiang Second People's Hospital of Southwest Medical University, 470 Xinjiang Road, Dongxing, Neijiang, Sichuan 641000, P.R. China

E-mail: jiang3w3@163.com

Key words: liver cancer, circ_0008537, miR-153-3p, MCL1, Snail1 liver cancer, hepatocellular carcinoma comprises more than $90 \%$ of all primary liver cancer cases (3). Liver cirrhosis is the predominant predisposing factor of liver cancer and usually occurs during chronic liver injury, resulting in subsequent death of liver cells (4). Common reasons of cirrhosis include metabolic liver disorders, autoimmune diseases, chronic viral infections and environmental factors (4). The incidence of liver cancer is unevenly distributed in the world due to the wide variability of the diverse pathogenic causes of cirrhosis. More than $80 \%$ of liver cancer cases are reported in Eastern Asia and Africa, where chronic hepatitis B viral infection is endemic (5). In contrast to hepatitis $B$, hepatitis $C$ viral infection is considered the most common cause of liver cancer in Europe and in the United States (6). In general, a variety of treatment methods that mainly include surgical resection and chemotherapy can effectively reduce tumor progression when the patients are diagnosed at the early stages, which increases considerably the five-year survival rate of the early-stage liver cancer patients (7). Unfortunately, a considerable number of liver cancer patients are diagnosed at the advanced stage of the disease, where the five-year survival rate remains dismal $(8,9)$. Understanding the etiology of liver cancer may be helpful in developing novel effective therapeutic targets for liver cancer patients.

As one of the most important members of non-coding RNAs, circular RNAs (circRNAs) have a unique circular structure that is completely different from other linear RNAs (10). A previous study has revealed that circRNAs are widely present in eukaryotic cells and play a key role during cell proliferation, apoptosis and differentiation (11). circRNAs exist mainly in the cytoplasm of eukaryotic cells and they act as sponges to specific microRNAs (miRNAs) in order to modulate the expression of target genes (12). Aberrant expression of circRNAs can be usually observed in pathological conditions, such as human cancers and neurodegenerative and autoimmune disorders (13-15). These aberrantly expressed circRNAs may be used as promising markers in disease screening. Manipulation of their expression is considered a popular research strategy in disease studies. In recent years, an increasing number of circRNAs have been identified that are dysregulated in liver cancer and play an important role during tumor growth as determined by in vitro and in vivo models, revealing the complex essence of liver carcinogenesis $(16,17)$. 
The present study aimed to investigate the biological functions and potential mechanisms of hsa_circ_0008537 (circ_0008537) in liver tumorigenesis. The data aimed to provide a novel therapeutic axis for liver cancer treatment.

\section{Materials and methods}

Liver cancer specimens and cell lines. A total of 70 liver cancer tissues and matched non-tumor samples were obtained from patients (aged 42-78 years; 39 male and 31 female patients) who were diagnosed with liver cancer at the Affiliated Neijiang Second People's Hospital of Southwest Medical University (Neijiang, China) from March 2009 to December 2019. The present study was approved (approval no. NSH-2019-005-020) by the Ethics Committee of the Affiliated Neijiang Second People's Hospital of the Southwest Medical University. The subjects provided written informed consent for their participation in the study protocol. The tissues were immersed into liquid nitrogen immediately following resection from the liver cancer patients and were subsequently transferred to $-80^{\circ} \mathrm{C}$ until further use. Four liver cancer cell lines (Huh-7, SNU449, SK-Hep-1 and HepG2), one normal immortalized liver cell line (THLE-3) and 293 cells were obtained from the American Type Culture Collection (ATCC) and maintained in $10 \%$ fetal bovine serum (FBS; product no. SH30406.05; HyClone; Cytiva) with Dulbecco's modified Eagle's medium (DMEM; product no. D5796; Sigma-Aldrich; Merck KGaA) at $37^{\circ} \mathrm{C}, 95 \% \mathrm{O}_{2}$ and $5 \% \mathrm{CO}_{2}$.

Reverse transcription-quantitative PCR (RT-qPCR). Total RNA was extracted from liver cancer tissues and cells following lysis using the TRIzol reagent (Invitrogen; Thermo Fisher Scientific, Inc.). Its quality was verified using spectrophotometric methods. Subsequently, $2 \mu \mathrm{g}$ RNA samples were subjected for reverse transcription with the Bestar ${ }^{\mathrm{TM}}$ qPCR RT kit (DBI Biosciences). To examine the resistance of circ_0008537 to RNase R, RT-qPCR was conducted on RNA with or without RNase R treatment. RT-qPCR was performed using specific divergent primers and the Power SYBR green master mix (Thermo Fisher Scientific, Inc.) on a 7500 Fast Real-Time PCR system (Applied Biosystems; Thermo Fisher Scientific, Inc.). The conditions of PRC amplification were enzyme activation at $95^{\circ} \mathrm{C}$ for $15 \mathrm{~min}$, followed by 40 cycles of denaturation at $95^{\circ} \mathrm{C}$ for $15 \mathrm{sec}$, annealing at $58^{\circ} \mathrm{C}$ for $30 \mathrm{sec}$, and extension at $72^{\circ} \mathrm{C}$ for $30 \mathrm{sec}$. The gene expression was quantitated using the $2^{-\Delta \Delta \mathrm{Cq}}$ method from 3 independent repetitions (18). The primers used in the present study are listed in Table I.

Sanger sequencing. As described in a previous study (19), the extracted total RNAs were used for Sanger sequencing, which was conducted by BGI Genomics Co., Ltd.

Oligonucleotide transfection. Two circ_0008537 siRNAs (si-circ-1 and si-circ-2), si-control (si-ctrl), circ_000853-overexpressed plasmids, empty vector (pcDNA3.1, EV), miR-153-3p mimic and its scramble control were all purchased from Integrated Biotech Solutions. Lipofectamine 3000 (Invitrogen; Thermo Fisher Scientific, Inc.), which were applied to complete the transfection of oligonucleotides. The sequences of the oligonucleotides included miR-153-3p mimic, 5'-UUGCAU AGUCACAAAAGUGAUC-3', scrambled miRNA scramble control, 5'-CAGUACUUUUGUGUAGUACAA-3'; si-circ-1 target sequences, 5'-AGCCCCACUACCAGGAUGAAA-3'; si-circ-2 target sequences, 5'-GAGCCCCACUACCAGGAU GAA-3'; si-ctrl, 5'-UCUGAGAGGAUUCUAGGU-3'. HepG2 or SK-Hep-1 cells were transfected with si-circ-1 (50 nM), si-circ-2 (50 nM), si-ctrl (50 nM), circ_0008537-overexpressed plasmids (50 ng), EV (pcDNA3.1, 50 ng), miR-153-3p mimics (100 nM) or scramble control $(100 \mathrm{nM})$ at $37^{\circ} \mathrm{C}$ for $48 \mathrm{~h}$ using Lipofectamine 3000 reagent (Invitrogen; Thermo Fisher Scientific, Inc.) based on the experimental instructions. After transfection for $48 \mathrm{~h}$, the cells were applied fort the subsequent experiments.

Colony formation assay. The treated liver cancer cells (2,000 cells/well) were seeded in 6-well plates containing $2 \mathrm{ml}$ DMEM and subsequently maintained in a cell incubator with $95 \% \mathrm{O}_{2}$ and $5 \% \mathrm{CO}_{2}$ for two weeks. Subsequently, the colonies were fixed in $75 \%$ ethanol at room temperature for $15 \mathrm{~min}$ and stained with Giemsa solution at room temperature for $20 \mathrm{~min}$. The visible colonies ( $>10$ cells) were counted manually and visualized by a light microscope using a magnification of $\mathrm{x} 20$.

EdU incorporation assay. The treated liver cancer cells $\left(1 \times 10^{4}\right.$ cells/well) were seeded into sterile 24 -well plates and the EdU kit (Invitrogen; Thermo Fisher Scientific, Inc.) was used to examine cell proliferation following the instructions of the manufacturer. Cell imaging was conducted using a laser confocal microscope (Olympus Corporation) with a magnification of $x 20$. DAPI $(1 \mathrm{mg} / \mathrm{ml})$ was utilized to stain the cell nucleus at room temperature for $10 \mathrm{~min}$.

Transwell assay. The Transwell chambers (8 $\mu \mathrm{m}$; Corning Inc.) were coated with or without Matrigel and were used to examine the abilities of invasion or migration, individually. The treated HepG2 and SK-Hep-1 cells ( $1 \times 10^{4}$ cells/well) were seeded into the upper chamber containing serum-free DMEM, and DMEM with FBS was placed into the lower chamber. Following $24 \mathrm{~h}$ of cell incubation, the cells invading or migrating the membrane were stained with $1 \%$ crystal violet at room temperature for $30 \mathrm{~min}$ after fixation in $4 \%$ paraformaldehyde at room temperature for $30 \mathrm{~min}$. Six random fields were selected for cell number counting using a light microscope at a magnification of $\mathrm{x} 20$.

Wound-healing assay. A total of $2 \mathrm{ml}$ DMEM containing treated liver cancer cells ( $1 \times 10^{5}$ cells) were added into $35-\mathrm{mm}$ petri dishes and cultured at $37^{\circ} \mathrm{C}$ until $100 \%$ confluence. Subsequently, a straight scratch was made by a sterile pipette tip $(100 \mu \mathrm{l})$ on the surface of the cell layer. The cells were allowed to grow at $37^{\circ} \mathrm{C}$ for an additional $24 \mathrm{~h}$. The images were obtained using an Olympus digital camera at the 0 - and 24-h time-points following cell scratching.

Bioinformatics. GLI3 was identified as the host gene of circ_0008537 through University of California Santa Cruz (UCSC) (http://genome.ucsc.edu/). The potential target miRNAs of circ_0008537 were predicted via starBase v2.0 (http:// starbase. sysu.edu.cn/) and circular RNA Interactome (https:/circinteractome.nia.nih.gov/). 
Table I. The sequences of primers in the RT-qPCR assay.

\begin{tabular}{ll}
\hline ID & \multicolumn{1}{c}{ Sequence (5'-3') } \\
\hline GAPDH & F: TGTTCGTCATGGGTGTGAAC \\
& R: ATGGCATGGACTGTGGTCAT \\
circ_0008537 & F: TTACATGGAGCCCCACTACC \\
miR-153-3p & R: TCGTCACTCGATGTTGAAGG \\
& F: GGCTCAAGTGTGATTCATACT \\
U6 & R: GAGTCAATACTCTTAAGGCATC \\
& F: CTTCGGCAGCACATATAC \\
& R: GAACGCTTCACGAATTTGC \\
\hline
\end{tabular}

RT-qPCR, reverse transcription-quantitative PCR; circ_00085377, hsa circ_0008537 miR-153-3p, microRNA-153-3p; F, forward; R, reverse.

Plasmid construction and dual luciferase activity assay. The wild-type (WT) or mutant (Mut) miR-153-3p binding sequence of circ_0008537 was inserted into the psi-CHECK-2 plasmid to establish the recombinant luciferase reporter plasmids (Luc-circ WT and Luc-circ Mut). 293 cells (ATCC) were co-transfected with Luc-circ WT or Luc-circ Mut and miR-153-3p or its scramble control with Lipofectamine 3000. Following $48 \mathrm{~h}$ of incubation after co-transfection, the firefly and Renilla luciferase activities of 293 cells were examined using the Dual-Luciferase Assay System (Promega Corporation) following the supplier's protocol. The relative luciferase activity was counted by normalizing the firefly luciferase activity against the Renilla luciferase activity.

RNA immunoprecipitation $(R I P)$. In line with the manufacturer's instructions, RIP was conducted using the EZMagna RIP kit (EMD Millipore). Briefly, liver cancer cells were lysed in RIPA lysis buffer (cat no. 89900; Thermo Fisher Scientific, Inc.) containing RNase repressor and the cell extracts were incubated with magnetic beads conjugated with Ago antibody (product code ab32381; Abcam) or IgG (product code ab207995; Abcam) overnight at $4^{\circ} \mathrm{C}$, after being stirred for $1 \mathrm{~h}$. Following washing with RIPA buffer three times, the RNA of the RNA/antibody complex was extracted using Trizol and its expression was analyzed using RT-qPCR.

Western blot analysis. Treated liver cancer cells were lysed in RIPA buffer (cat no. 89900; Thermo Fisher Scientific, Inc.) for total protein extraction. The protein concentration was determined using a BCA kit (Beyotime Institute of Biotechnology) and the protein samples $(40 \mu \mathrm{g})$ in each lane were separated with $10 \%$ SDS-PAGE. The target proteins were transferred to a PVDF membrane (EMD Millipore), which was incubated in 5\% non-fat milk at room temperature for 90 min to block non-specific binding sites. Subsequently, the membranes were incubated at $4^{\circ} \mathrm{C}$ overnight with primary antibodies against pro-survival protein myeloid cell leukemia 1 (MCL1; rat product code ab243136; 1:1,000), Snail1 (rabbit; 1:1,000; product code ab216347) and actin (rabbit; product code ab179467; 1:5,000; all from Abcam). The membranes were probed with HRP-conjugated donkey anti-rabbit secondary antibodies (1:2,000; product code ab6802; Abcam) at room temperature for $1 \mathrm{~h}$. The signals were visualized using an ECL system (Thermo Fisher Scientific, Inc.).

Kyoto encyclopedia of genes and genomes (KEGG) analysis and ceRNA network. The biological pathways of miR-153-3p were analyzed using KEGG (http://www.genome. jp/kegg/) (20). The circ_0008537-miR-153-3p-mRNA regulatory network was established using the Cytoscape software (Version 3.4.0, http://www. cytoscape.org/).

Statistical analysis. The data are presented as the mean \pm SEM. The data were statistically analyzed using SPSS 21.0 (IBM Corp.) and the statistical graphs were drawn using GraphPad Prism (version 8; GraphPad Prism Software, Inc.). Paired t-tests were employed to analyze the statistical significance between tumor and non-tumor samples, and unpaired t-tests were used for the comparison of the other two groups. For non-parametric, data in which the medians were compared, Wilcoxon rank-sum test was used for paired samples and Mann-Whitney U test for unpaired samples. One-way ANOVA with post hoc Tukey's test was applied for the multiple comparisons. The correlations were analyzed using Pearson correlation analysis. Kaplan-Meier curves and the log-rank test were applied for the differences in survival. $\mathrm{P}<0.05$ was considered to indicate a statistically significant difference.

\section{Results}

circ_00085377 is highly expressed in liver cancer. A previous study has demonstrated using microarray analysis that clusters of circRNAs are aberrantly expressed in liver cancer compared with the corresponding expression in adjacent non-tumorous tissues (21). Among these dysregulated circRNAs, the expression levels of 24 circRNAs were upregulated in liver cancer samples compared to the corresponding levels noted in normal samples. These circRNAs were widely distributed in chromosomes 1-7 and 11-19 among which circ_0008537 exhibited the highest-fold changes with regard to differential expression (Fig. 1A). By searching UCSC, GLI3 was identified as the host gene of circ_0008537, whereas the splicing site of circ_0008537 was determined by Sanger sequencing (Fig. 1B). Convergent and divergent primers were adopted to amplify circ_0008537 based on cDNA or gDNA in the presence of RNase R. The results indicated that circ_0008537 could be amplified by divergent primers based on cDNA but not on gDNA (Fig. 1C). Moreover, RNase R exposure degraded linear GLI3, while no effects were observed on the expression of circ_0008537 in SK-Hep-1 and HepG2 cells (Fig. 1D and E). RT-qPCR analysis indicated higher expression levels of circ_0008537 in liver cancer tissue samples than those observed in matched non-tumor samples $(\mathrm{P}<0.001$; Fig. 1F). Moreover, the data indicated that circ_0008537 expression was increased in stage III-IV compared with that observed in stage I-II tumors $(\mathrm{P}<0.001$; Fig. 1G). In addition, four liver cancer cell lines (Huh-7, SNU449, SK-Hep-1 and HepG2) and one normal immortalized liver cell line (THLE-3) were used for circ_0008537 level detection. The results demonstrated that circ_0008537 expression was significantly 

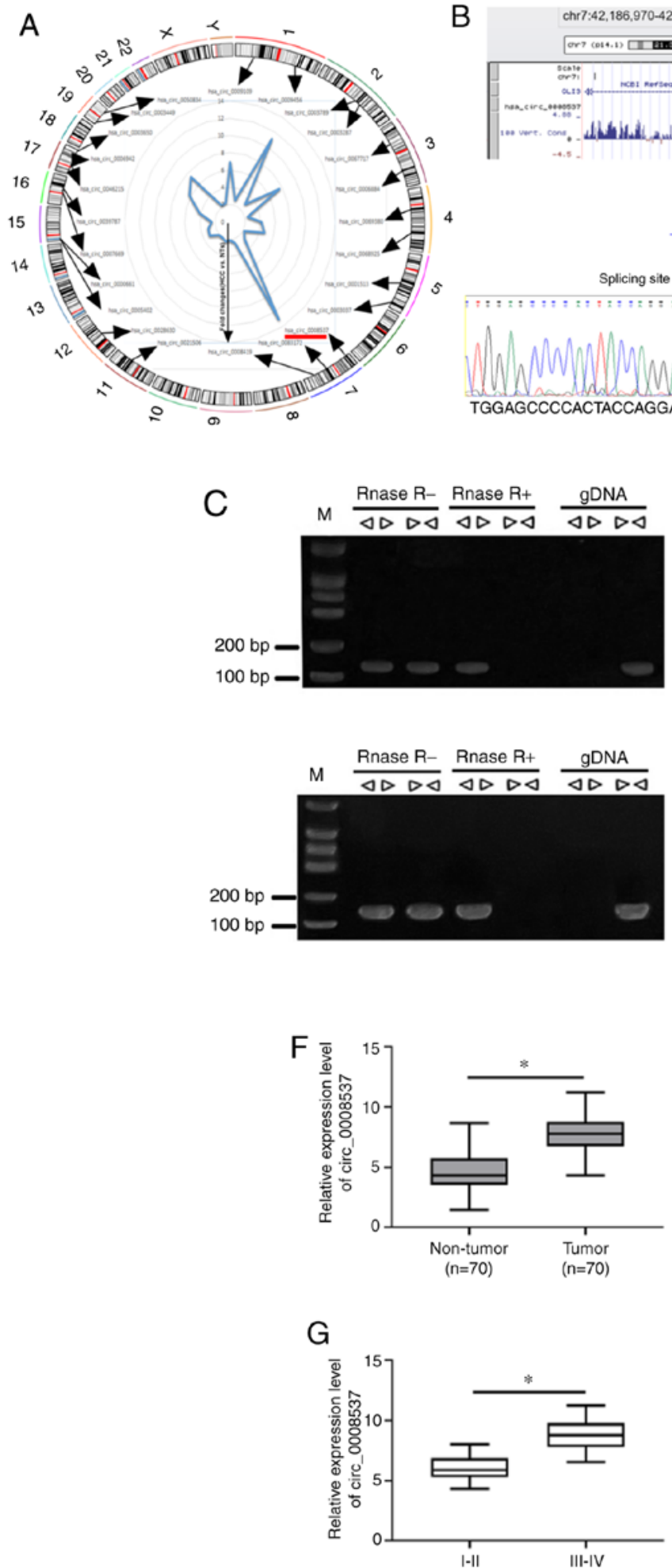
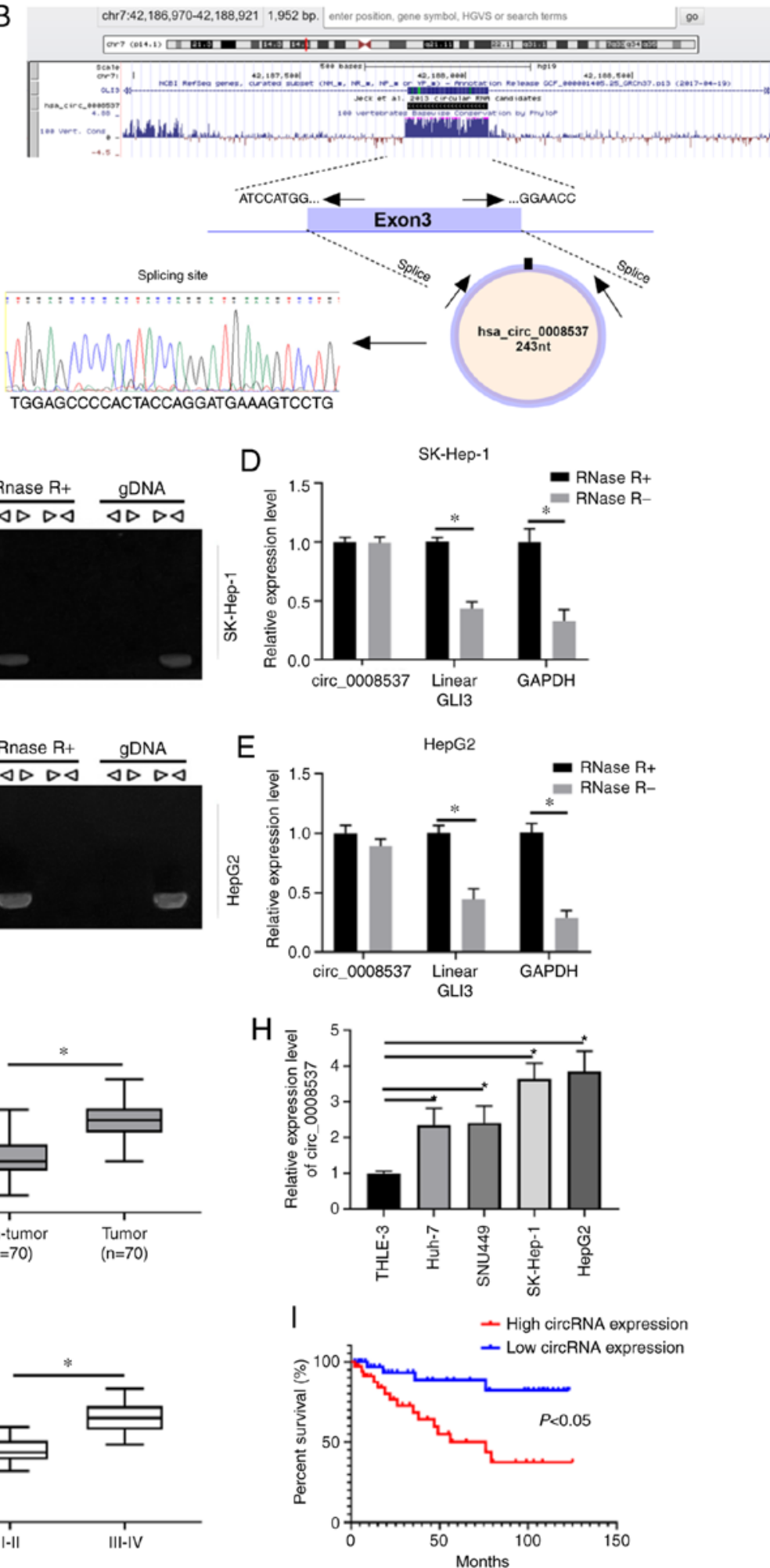

Figure 1. circ 00085377 is highly expressed in liver cancer. (A) Distribution of circRNAs per chromosome and their expression-fold changes in liver cancer patients compared to the corresponding levels of the healthy control subjects. (B) The schematic of circ_0008537 formation, and its validation through Sanger sequencing. (C-E) RT-qPCR validation of linear GLI3 mRNA and circ_0008537 in SK-Hep-1 and HepG2 cells in the presence and/or absence of RNase R. (F and G) Relative expression of circ_0008537 in 70 pairs of liver tumor and non-tumor tissues, as well as in tumors with different clinical stages. (H) Expression of circ_0008657 in four liver cancer cell lines (Huh-7, SNU449, SK-Hep-1 and HepG2). The THLE-3 cell line was used as a control. (I) Survival rate of liver cancer patients with high and low expression of circ_0008537. "P<0.05. circ_00085377, hsa_circ_0008537; circRNAs, circular RNAs; RT-qPCR, reverse transcription-quantitative PCR.

upregulated in Huh-7, SNU449, SK-Hep-1 and HepG2 cell lines compared with the expression observed in the THLE-3 cell line $(\mathrm{P}<0.01$ and $\mathrm{P}<0.001$; Fig. $1 \mathrm{H})$. The analysis of the overall survival demonstrated that liver cancer patients with high expression of circ_0008537 exhibited a worse survival rate than those with low expression of circ_0008537 ( $\mathrm{P}<0.05$; 

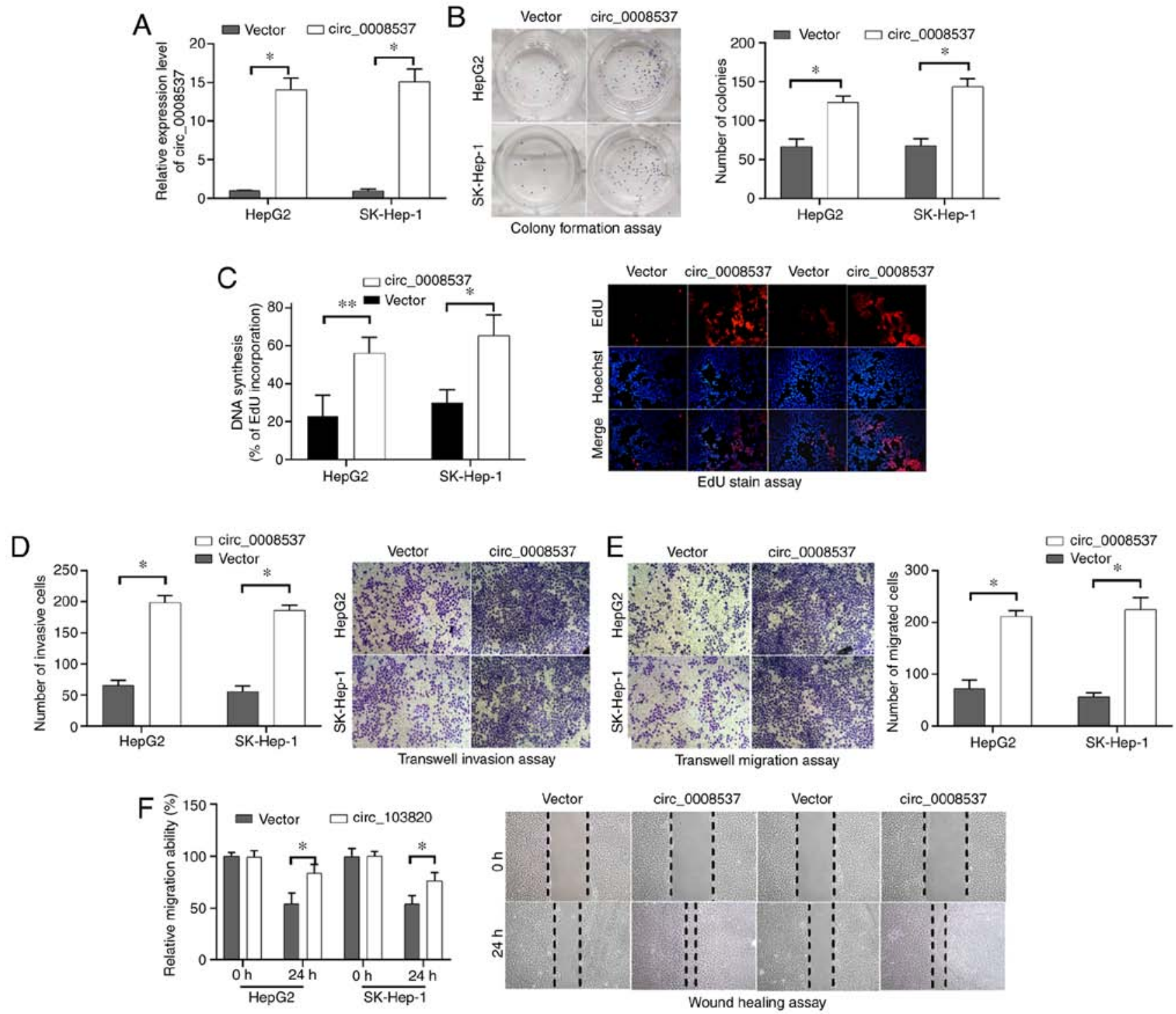

Figure 2. circ_0008537 overexpression facilitates liver cancer cell proliferation, invasion and migration. (A) RT-qPCR assessment of circ_0008537 expression in HepG2 and SK-Hep-1 cells transfected with vector and circ_0008537. HepG2 and SK-Hep-1 cells were transfected with circ_0008537 or empty vector and cultured for $24 \mathrm{~h}$. Subsequently, cell proliferation was assessed using (B) colony formation and (C) EdU staining, whereas cell invasion was analyzed using (D) the Transwell invasion assay and cell migration by (E) Transwell cell migration assay and (F) wound-healing assays. ${ }^{*} \mathrm{P}<0.05$ and ${ }^{* * *} \mathrm{P}<0.01$. circ_00085377, hsa_circ_0008537; RT-qPCR, reverse transcription-quantitative PCR.

Fig. 1I). These findings indicated that circ_0008537 expression was increased in liver cancer and that it was associated with a worse prognosis.

circ_0008537 overexpression facilitates liver cancer cell proliferation invasion and migration. To investigate the function of circ_0008537 in livercancer, the effects of circ_0008537 overexpression on liver cancer cell proliferation, invasion and migration were investigated in vitro. Following transfection of circ_0008537, its expression levels were significantly increased in HepG2 and SK-Hep-1 cells compared to those observed in cells transfected with empty vector $(\mathrm{P}<0.001$; Fig. $2 \mathrm{~A})$. The colony formation assay demonstrated that circ_0008537 overexpression significantly increased the number of HepG2 and SK-Hep-1 cell colonies $(\mathrm{P}<0.01$; Fig. $2 \mathrm{~B})$, indicating that liver cancer cell proliferation was induced by circ_0008537 overexpression. The results derived from EdU staining further confirmed this conclusion $(\mathrm{P}<0.01$; Fig. $2 \mathrm{C})$. By using
Transwell chambers coated with or without Matrigel, the experiments demonstrated that circ_0008537 overexpression facilitated the invasive and migratory abilities of HepG2 and SK-Hep-1 cells ( $\mathrm{P}<0.001$; Fig. 2D and E). The effects of circ_0008537 overexpression on liver cancer cell migratory ability were further supported by the wound-healing assay in HepG2 and SK-Hep-1 cells ( $\mathrm{P}<0.05$; Fig. 2F). The results indicated that circ_0008537 overexpression facilitated liver cancer cell proliferation, invasion and migration in vitro.

circ_0008537 silencing suppresses liver cancer cell proliferation, invasion and migration. circ_0008537 silencing was carried out in HepG2 and SK-Hep-1 cells to further study its functions in liver cancer cell proliferation, invasion and migration. circ_0008537 expression was significantly decreased in HepG2 and SK-Hep-1 cells following transfection of siRNAs against circ_0008537 (si-circ-1 and si-circ-2) compared with that observed in the si-control group ( $\mathrm{P}<0.05$; Fig. $3 \mathrm{~A})$. 
A

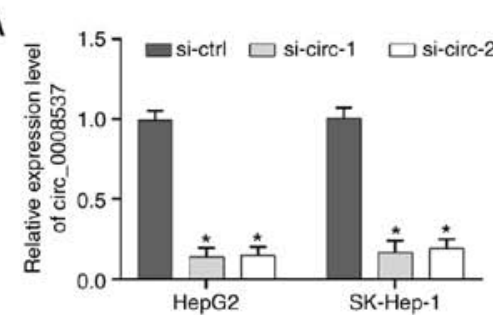

D
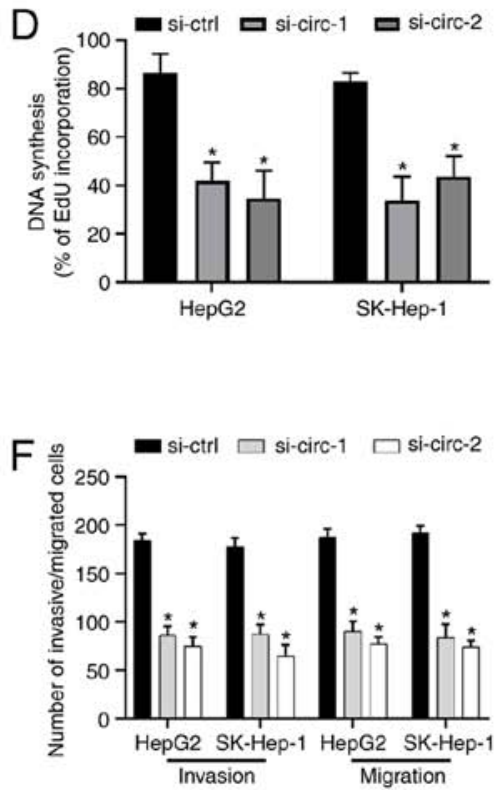
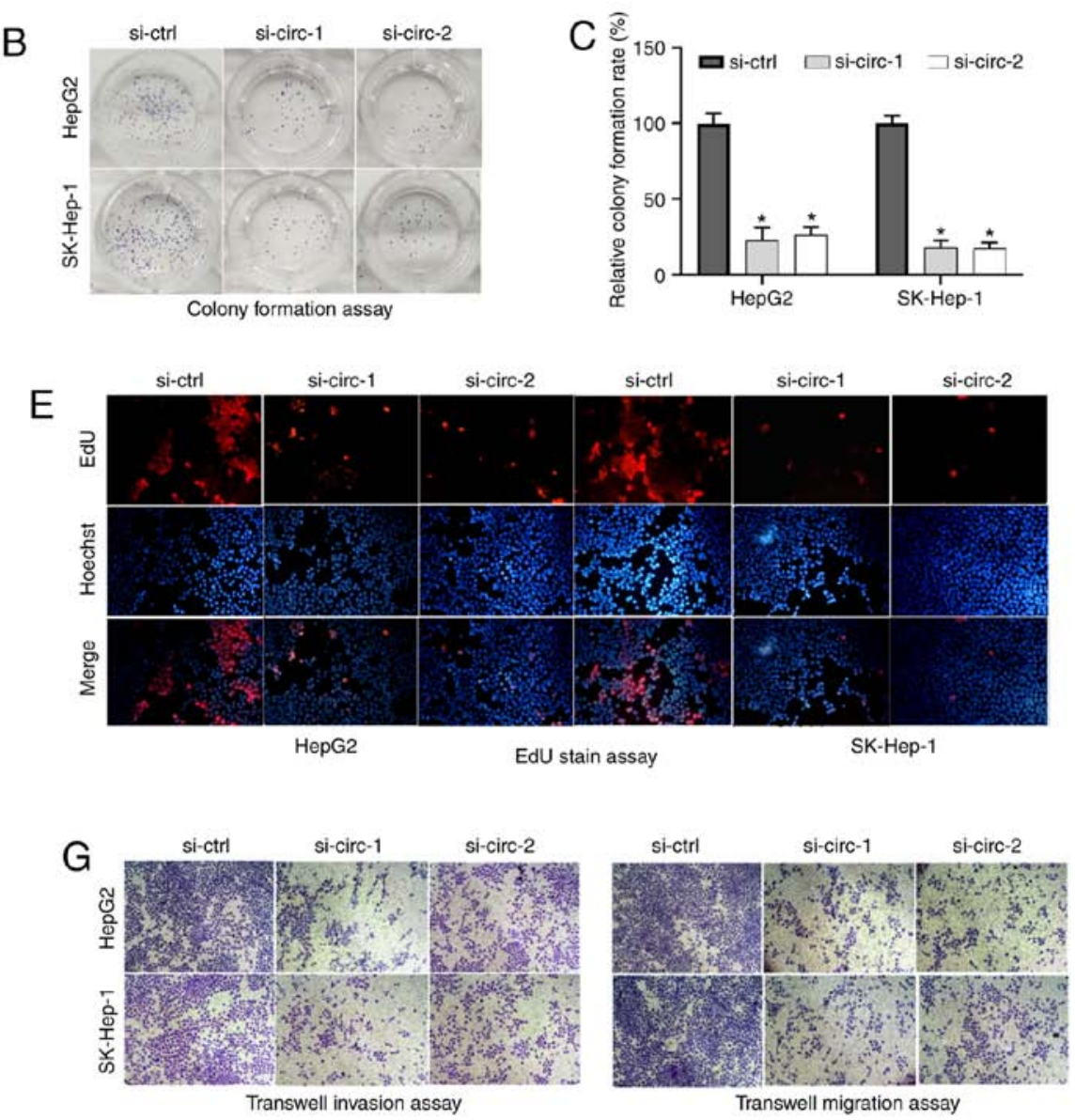

Figure 3. circ_0008537 silencing suppresses liver cancer cell proliferation, invasion and migration. (A) RT-qPCR assessment of circ_0008537 expression in HepG2 and SK-Hep-1 cells transfected with si-control and two siRNAs against circ_0008537 (si-circ-1 and si-circ-2). Cell proliferation of HepG2 and SK-Hep-1 cells was estimated by (B and C) colony formation and (D and E) EdU staining following circ_0008537 knockdown. (F and G) The invasive and migratory abilities of HepG2 and SK-Hep-1 cells were evaluated by Transwell assays following circ_0008537 knockdown. ${ }^{*} \mathrm{P}<0.05$. circ_00085377, hsa_circ_0008537; RT-qPCR, reverse transcription-quantitative PCR.

By using the colony formation assay and EdU staining, the analysis demonstrated that circ_0008537 silencing resulted in a significant inhibition of cell proliferative activity in HepG2 and SK-Hep-1 cells $(\mathrm{P}<0.01$ and $\mathrm{P}<0.01$; Fig. 3B-E). In addition, circ_0008537 silencing significantly attenuated the invasive and migratory activities of HepG2 and SK-Hep-1 cells $(\mathrm{P}<0.001$; Fig. 3F and $\mathrm{G})$. Therefore, circ_0008537 silencing repressed liver cancer cell proliferation, invasion and migration in vitro.

circ_0008537 serves as a sponge of $\mathrm{miR}-153-3 p$. To explore the mechanism of circ_0008537 in liver carcinogenesis, starBase and Circular RNA Interactome were used to screen the potential target miRNAs of circ_0008537. Only miR-153-3p and miR-331-3p were predicted by both the starBase and the Circular RNA Interactome (Fig. 4A). Dual-luciferase assay was adopted to validate the interplay between miR-151-3p and circ_0008537. Transfection of the cells with miR-153-3p caused a significant reduction of luciferase intensity of 293 cells driven by wild-type circ_0008537 (Luc_Circ WT), while these effects were not observed in 293 cells when the binding sequence was mutated $(\mathrm{P}<0.001$; Fig. 4B and C). Since Ago2 is an essential protein in the formation of RNA-induced silencing complex (RISC) (22), an RNA-binding protein immunoprecipitation assay was performed using Ago2 to further assess whether miR-153-3p and circ_0008537 could form a RISC. circ_0008537 was enriched by miR-153-3p in the presence of Ago2 ( $\mathrm{P}<0.001$; Fig. 4D and E). A negative correlation was observed in the expression levels of miR-153-3p and circ_0008537 in liver cancer $(\mathrm{P}<0.001$; Fig. $4 \mathrm{~F})$. In addition, the relative expression of $\mathrm{miR}-153-3 \mathrm{p}$ in liver cancer tissue samples was revealed to be decreased compared with matched normal samples $(\mathrm{P}<0.001$; Fig. 4G). Overall, the data indicated that circ_0008537 served as a sponge of miR-153-3p and revealed a negative correlation with the expression of the latter in liver cancer.

miR $-153-3 p$ reverses the facilitated effects of circ_000857 on liver cancer cell proliferation, invasion and migration. Subsequently, a rescue assay was performed to investigate whether miR-153-3p was involved in liver cancer progression mediated by circ_0008537. RT-qPCR analysis indicated that miR-153-3p transfection reversed the downregulation of miR-153-3p expression induced by circ_0008537 overexpression in both HepG2 and SK-Hep-1 cells $(\mathrm{P}<0.05$; Fig. 5A). The colony formation assay demonstrated that the circ_0008537 overexpression-induced effects on cell proliferation were abolished by miR-153-3p transfection $(\mathrm{P}<0.01$ 
A

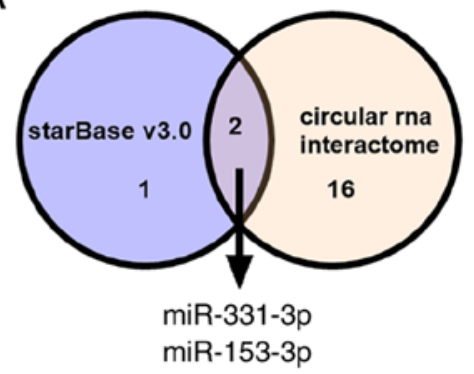

B

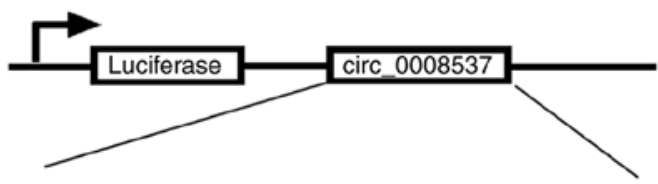

circ_0008537 WT 5'-GAAGAAACGCAAUCACUAUGCAG-3' | $|\||\||$ miR-153-3p 3'-CUAGUGAAAACACUGAUACGUU-5' circ_0008537 Mut 5'-GAAGAAACGCAAUCACCCGCAGG-3'
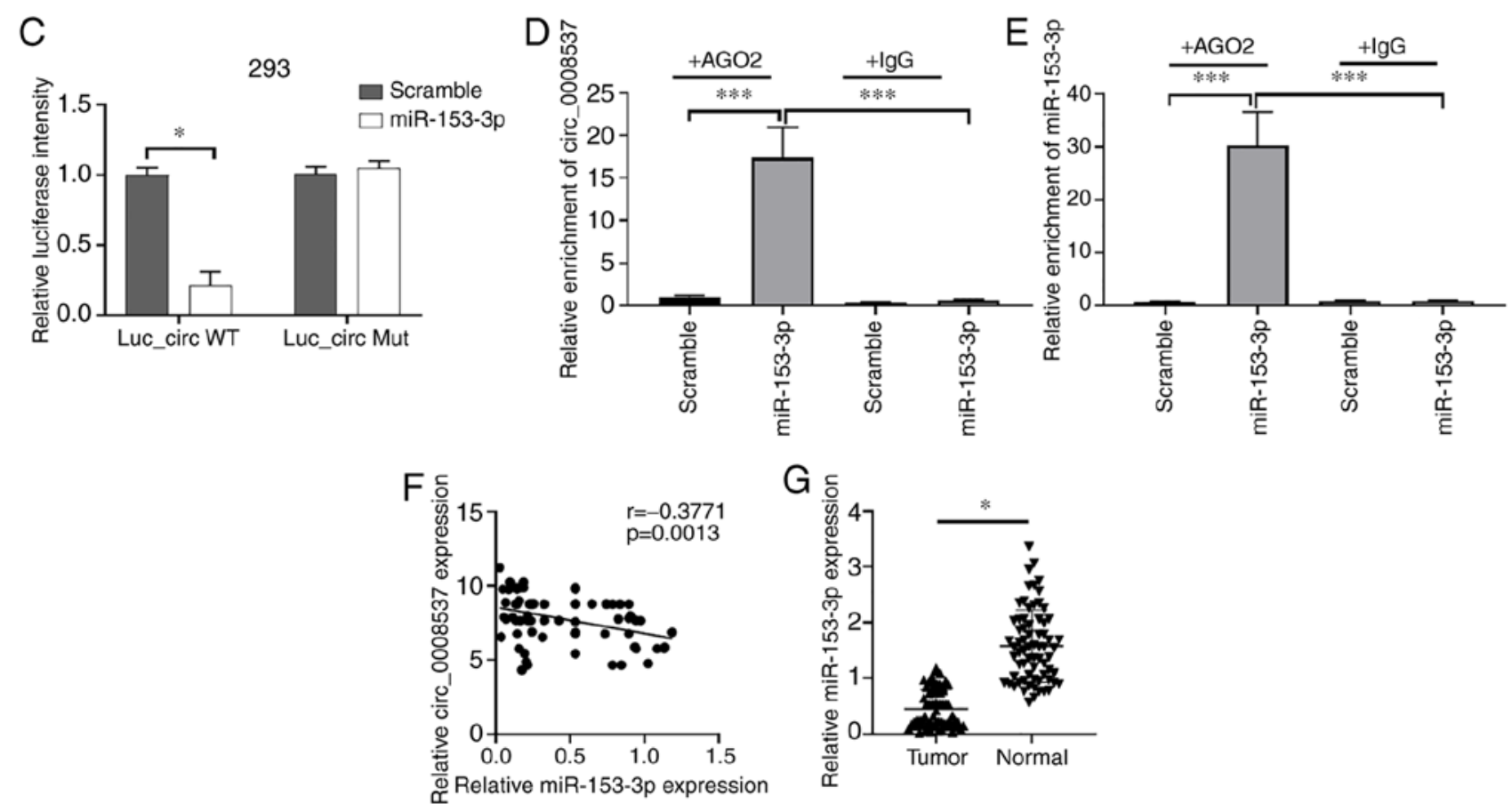

Figure 4. circ_0008537 serves as a sponge of miR-153-3p. (A) Venn diagram revealing the intersection of target miRNAs of circ_0008537 predicted by starBase and circular RNA interactome. (B) The sequence of WT and Mut circ_0008537. (C) The interaction between circ_0008537 and miR-153-3p was validated by dual-luciferase reporter experiments in 293 cells. (D and E) RNA immunoprecipitation was carried out to assess the interaction between circ_0008537 and miR-153-3p using anti-AGO2 antibody or control IgG in HepG2 and SK-Hep-1 cells. (F) Association between the expression levels of circ_0008537 and miR-153-3p in liver cancer $(\mathrm{r}=-0.3771, \mathrm{P}=0.0013)$. $(\mathrm{G})$ The mean expression levels of miR-153-3p noted in 70 liver cancer tissues and their corresponding adjacent controls. ${ }^{*} \mathrm{P}<0.05$ and ${ }^{* * *} \mathrm{P}<0.001$. circ_00085377, hsa_circ_0008537; miRNAs, micro RNAs; WT, wild-type; Mut, mutant; miR-153-3p, microRNA-153-3p.

and $\mathrm{P}<0.001$; Fig. 5B and C). The enhanced migratory and invasive abilities in the circ_0008537-overexpressed HepG2 and SK-Hep-1 cells were abrogated by miR-153-3p $(\mathrm{P}<0.05$; Fig. 5D and E). Overall, miR-153-3p reversed the facilitated effects of circ_000857 on liver cancer cell proliferation, invasion and migration.

circ_0008537 indirectly regulates MCL1 and Snail1 through miR-153-3p. The bioinformatic analysis predicted multiple target genes of miR-153-3p, including MCL1 and Snaill. In order to investigate whether MCL1 and Snail1 were regulated by miR-153-3p, their expression levels were investigated by western blotting following overexpression of miR-153-3p. The results indicated that miR-153-3p overexpression significantly decreased the expression levels of MCL1 and Snail1 in HepG2 and SK-Hep-1 cells, while circ_0008537 overexpression exhibited the opposite effects (Fig. 6A). In view of the interplay between circ_0008537 and miR-153-3p in liver cancer, further investigations examined whether the regulatory effects of circ_0008537 on MCL1 and Snail1 could be abolished by miR-153-3p. Co-transfection of circ_0008537 and miR-153-3p reversed the downregulation effect on MCL1 and Snaill induced by miR-153-3p overexpression (Fig. 6B). KEGG pathway analysis was subsequently performed on the identified target genes of miR-153-3p. The top ten pathways of miR-153-3p are presented in Fig. 6C. A ceRNA network indicated the interplay of circ_0008537 with miR-153-3p and its target genes (Fig. 6D). Overall, the data demonstrated that circ_0008537 indirectly regulated the expression levels of MCL1 and Snail1 through miR-153-3p (Fig. 6E).

\section{Discussion}

In view of the high possibility of tumor metastasis, liver cancer has long been considered an aggressive malignant tumor, which reduces the life expectancy of the affected population (23). The understanding of the potential molecular mechanisms of liver cancer pathogenesis has long been considered the most effective strategy of developing drugs for 

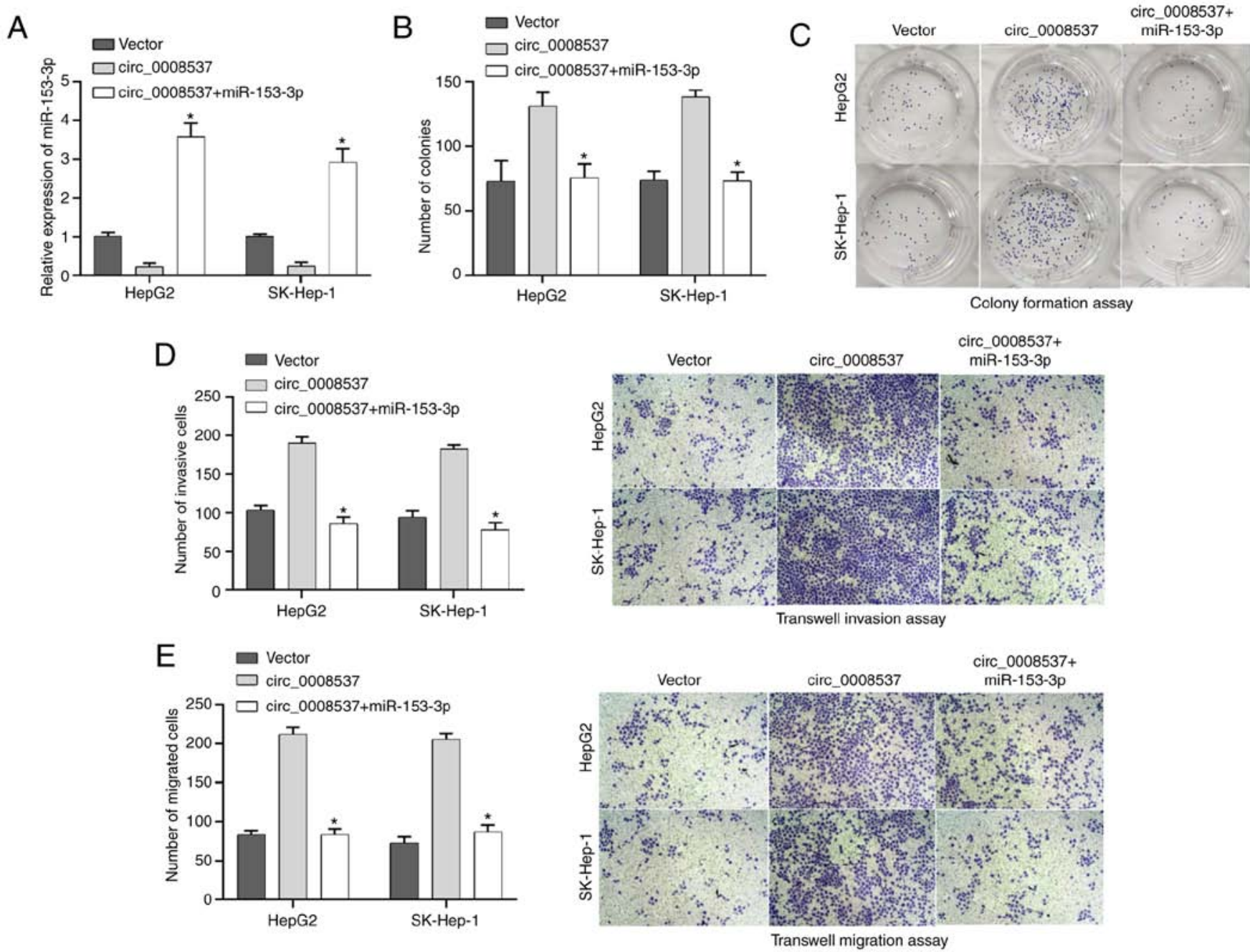

Figure 5. miR-153-3p reverses the facilitated effects of circ_000857 on liver cancer cell proliferation, invasion and migration. (A) RT-qPCR analysis of miR-153-3p was performed in HepG2 and SK-Hep-1 cells following co-transfection of circ_0008537 and miR-153-3p. (B and C) Colony formation was employed to analyze the cell proliferation of HepG2 and SK-Hep-1 cells following $24 \mathrm{~h}$ of incubation after transfection of circ_0008537 and miR-153-3p. Cell (D) invasion and (E) migration was assessed by Transwell assays in HepG2 and SK-Hep-1 cells transfected with circ_0008537 and miR-153-3p. ${ }^{*} \mathrm{P}<0.05$ vs. circ_0008537 group. miR-153-3p, microRNA-153-3p; circ_00085377, hsa_circ_0008537; RT-qPCR, reverse transcription-quantitative PCR.

liver cancer treatment. Nevertheless, only a limited number of effective drugs have been developed due to the complexity of liver cancer etiology (24). circRNAs have quickly become one of the main research focuses in the field of liver cancer since they were initially reported to be involved in liver tumorigenesis. In the present study, differentially expressed circRNAs were screened between liver cancer and matched normal tissue samples. The investigations focused on the role and mechanisms of circ_0008537 increased expression levels in liver cancer tumor growth.

The Hedgehog (Hh) signaling pathway, an extremely conserved pathway in evolution, has been reported to be involved in multiple aspects of embryonic development and stem cell maintenance (25). Recently, the Hh pathway was revealed to be abnormally activated in various types of malignancies, including non-small cell lung, esophageal and liver cancers (26-28). As a key mediator of the Hh cascade, Gli3 has also been revealed to act as a tumor promoter in human tumors (29). Gli3 inhibition by miR-378 was reported to attenuate activation of hepatic stellate cells and liver fibrosis (30). In the present study, circ_0008537 interacted with the GLI3 gene and was highly expressed in liver cancer tissues and cells.

The role of circRNAs in liver tumorigenesis has been well documented by accumulating evidence, indicating both oncogenic and inhibitory tumor progression effects (16). circHIAT1 has been identified as an oncogene in liver cancer by modulating the PTEN pathway via sponging miR-3171 (31). In contrast to circHIAT1, circRNA-ABCB10 was confirmed to be a liver cancer repressor by sponging miR-340-5p and miR-452-5p to release NRP1 and ABL2 (32). The adverse effects of different circRNAs in the pathogenesis of liver cancer indicate the complex interactions of circRNAs with pathways that lead to the development of liver cancer. In the present study, overexpression and silencing experiments of circ_0008537 were performed in liver cancer cells and the data demonstrated that circ_0008537 acted as an oncogene in liver cancer. To the best of our knowledge, the present study is the first to report the biological functions of circ_0008537 in liver tumorigenesis.

miRNAs are a class of noncoding single stranded RNA molecules of approximately 22 nucleotides in length, which 


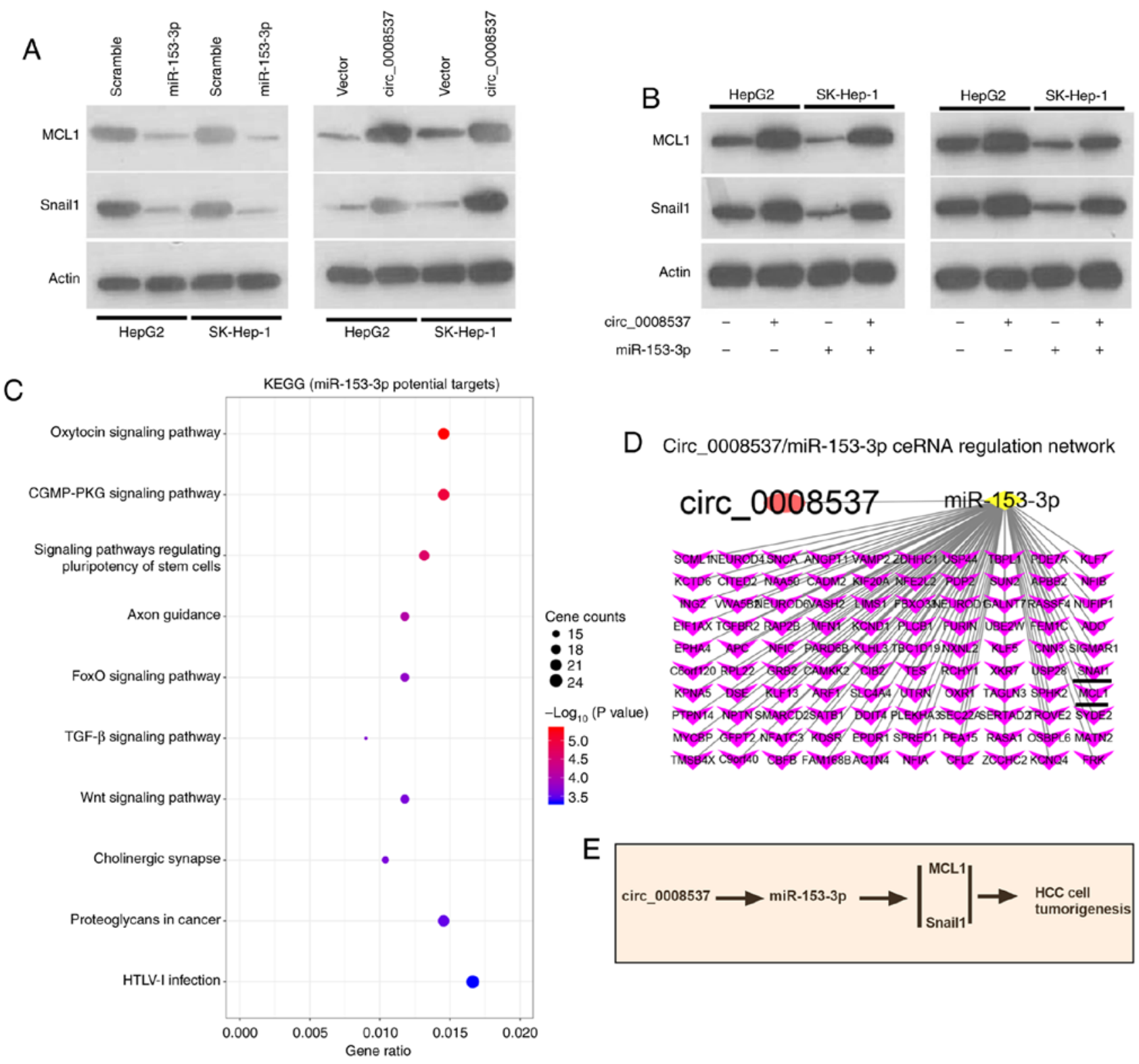

Figure 6. circ_0008537 indirectly regulates MCL1 and Snail1 through miR-153-3p. (A) Western blot analysis was conducted in HepG2 and SK-Hep-1 cells transfected with miR-153-3p or circ_0008537 alone to detect the expression levels of MCL1 and Snaill. (B) Following co-transfection of HepG2 and SK-Hep-1 cells with circ_0008537 and miR-153-3p, western blot analysis was performed for the detection of MCL1 and Snaill expression. (C) KEGG analysis of miR-153-3p potential target genes. (D) Circ_0008537/miR-153-3p ceRNA regulatory network. (E) The diagram of the mechanisms underlying the circ_0008537/miR-153-3p axis in liver cancer. circ_00085377, hsa_circ_0008537; miR-153-3p, microRNA-153-3p; KEGG, Kyoto Encyclopedia of Genes and Genomes.

are involved in a range of crucial biological processes, including development, hematopoiesis, organogenesis, apoptosis, proliferation and even cancer development $(33,34)$. The miRNA-mRNA axis has been demonstrated by numerous studies to be one of the most common pathways through which circRNAs play their roles in regulating tumor growth at the transcriptional and post transcriptional levels $(14,35)$. Therefore, the potential target miRNAs of circ_0008537 were predicted using the following two bioinformatic software: starBase and circular RNA interactome. Two miRNAs (miR-153-3p and miR-331-3p) were identified and miR-153-3p was selected for further analysis. miR-153-3p has been reported to participate in tumor progression of multiple human tumors, such as gastric cancer, esophageal squamous cell carcinoma and oral squamous cell carcinoma (36-38).
Nevertheless, the role of miR-153-3p in liver cancer has not been previously investigated. In the present study, a negative correlation between the expression levels of miR-153-3p and circ_0008537 was revealed. Overexpression of miR-153-3p could reverse the tumor promoting effects of circ_0008537 on liver cancer progression. Moreover, miR-153-3p was revealed to abolish the downregulation of MCL1 and Snaill expression induced by miR-153-3p.

In conclusion, the present study provided in vitro evidence demonstrating that circ_0008537 facilitated liver carcinogenesis by indirectly regulating MCL1 and Snaill through miR-153-3p. However, in vivo studies are required to further confirm this conclusion. In addition, we will explore the more detailed mechanisms of the circ_0008537/miR-153-3p axis in HCC progression in a future study. 


\section{Acknowledgements}

Not applicable.

\section{Funding}

The present work was supported by The Sichuan Provincial Department of Science and Technology of China (grant no. 15089), the Sichuan Province Department of Medical Association Scientific Research Subject of China (grant no. S16043), the Youth Innovation of Scientific Research Subject of Sichuan Province Medical Association of China (grant no. 2016, Q16044), the Scientific Research Subject of Science and Technology Bureau of Sichuan Province Neijiang City of China (grant no. Neijiang 2017021), and the Planning Project of Sichuan Provincial Science and Technology Department (grant no. 2018JY0419).

\section{Availability of data and materials}

The datasets used and/or analyzed during the current study are available from the corresponding author on reasonable request.

\section{Authors' contributions}

GY and OJ designed the experiments. GY, OJ, XL, JL, SH, YW, JZ and DL performed the experiments. GY, XL, JL, SH collected the data. OJ and JZ analyzed the data. OJ provided the resource supports. GY, XL and OJ drafted the manuscript. All authors read and approved the final manuscript.

\section{Ethics approval and consent to participate}

The present study was approved (approval no. NSH-2019005-020) by the Ethics Committee of the Affiliated Neijiang Second People's Hospital of the Southwest Medical University. The subjects provided written informed consent for their participation in the study protocol.

\section{Patient consent for publication}

Not applicable.

\section{Competing interests}

The authors have no commercial or other associations that may pose a conflict of interest.

\section{References}

1. Kudo M, Kitano M, Sakurai T and Nishida N: General rules for the clinical and pathological study of primary liver cancer, nationwide follow-up survey and clinical practice guidelines: The outstanding achievements of the liver cancer study group of Japan. Dig Dis 33: 765-770, 2015.

2. Siegel RL, Miller KD and Jemal A: Cancer statistics, 2020 CA Cancer J Clin 70: 7-30, 2020.

3. Lafaro KJ, Demirjian AN and Pawlik TM: Epidemiology of hepatocellular carcinoma. Surg Oncol Clin N Am 24: 1-17, 2015

4. Moradpour D and Blum HE: Pathogenesis of hepatocellular carcinoma. Eur J Gastroenterol Hepatol 17: 477-483, 2005.

5. El-Serag HB: Hepatocellular carcinoma. N Engl J Med 365: 1118-1127, 2011
6. Chonprasertsuk S and Vilaichone RK: Epidemiology and treatment of hepatocellular carcinoma in Thailand. Jpn J Clin Oncol 47: 294-297, 2017.

7. Levrero M: Viral hepatitis and liver cancer: The case of hepatitis C. Oncogene 25: 3834-3847, 2006.

8. Mazzoccoli G, Miele L, Oben J, Grieco A and Vinciguerra M: Biology, epidemiology, clinical aspects of hepatocellular carcinoma and the role of sorafenib. Curr Drug Targets 17: 783-799, 2016.

9. Worns MA and Galle PR: Hepatocellular carcinoma in 2017: Two large steps forward, one small step back. Nat Rev Gastroenterol Hepatol 15: 74-76, 2018.

10. Patop IL and Kadener S: circRNAs in cancer. Curr Opin Genet Dev 48: 121-127, 2018

11. Yu T, Wang Y, Fan Y, Fang N, Wang T, Xu T and Shu Y: CircRNAs in cancer metabolism: A review. J Hematol Oncol 12: 90, 2019.

12. Szabo L and Salzman J: Detecting circular RNAs: Bioinformatic and experimental challenges. Nat Rev Genet 17: 679-692, 2016.

13. Haque S and Harries LW: Circular RNAs (circRNAs) in health and disease. Genes (Basel) 8: 353, 2017.

14. Han B, Chao J and Yao H: Circular RNA and its mechanisms in disease: From the bench to the clinic. Pharmacol Ther 187: 31-44, 2018.

15. Akhter R: Circular RNA and Alzheimer's disease. Adv Exp Med Biol 1087: 239-243, 2018.

16. Fu L, Jiang Z, Li T, Hu Y and Guo J: Circular RNAs in hepatocellular carcinoma: Functions and implications. Cancer Med 7: 3101-3109, 2018

17. Hu J, Li P, Song Y, Ge YX, Meng XM, Huang C, Li J and Xu T: Progress and prospects of circular RNAs in hepatocellular carcinoma: Novel insights into their function. J Cell Physiol 233: 4408-4422, 2018.

18. Livak KJ and Schmittgen TD: Analysis of relative gene expression data using real-time quantitative PCR and the 2(-Delta Delta C(T)) method. Methods 25: 402-408, 2001.

19. Zheng J, Zhang H, Banerjee S, Li Y, Zhou J, Yang Q, Tan X, Han P, Fu Q, Cui X, et al: A comprehensive assessment of next-generation sequencing variants validation using a secondary technology. Mol Genet Genomic Med 7: e00748, 2019.

20. Wang Z, Shang P, Li Q, Wang L, Chamba Y, Zhang B, Zhang H and $\mathrm{Wu}$ C: iTRAQ-based proteomic analysis reveals key proteins affecting muscle growth and lipid deposition in pigs. Sci Rep 7: 46717, 2017

21. Cui S, Qian Z, Chen Y, Li L, Li P and Ding H: Screening of upand downregulation of circRNAs in HBV-related hepatocellular carcinoma by microarray. Oncol Lett 15: 423-432, 2018.

22. Chen Y, Yang F, Fang E, Xiao W, Mei H, Li H, Li D, Song H, Wang J, Hong M, et al: Circular RNA circAGO2 drives cancer progression through facilitating HuR-repressed functions of AGO2-miRNA complexes. Cell Death Differ 26: 1346-1364, 2019.

23. Blum HE: Molecular therapy and prevention of hepatocellular carcinoma. Hepatobiliary Pancreat Dis Int 2: 11-22, 2003.

24. Grandhi MS, Kim AK, Ronnekleiv-Kelly SM, Kamel IR, Ghasebeh MA and Pawlik TM: Hepatocellular carcinoma: From diagnosis to treatment. Surg Oncol 25: 74-85, 2016.

25. Skoda AM, Simovic D, Karin V, Kardum V, Vranic S and Serman L: The role of the Hedgehog signaling pathway in cancer: A comprehensive review. Bosn J Basic Med Sci 18: 8-20, 2018.

26. Zhuang H, Cao G, Kou C and Liu T: CCL2/CCR2 axis induces hepatocellular carcinoma invasion and epithelial-mesenchymal transition in vitro through activation of the Hedgehog pathway. Oncol Rep 39: 21-30, 2018.

27. Liu Y,Huber RM, Kiefl R, Tufman A and Kauffmann-Guerrero D: Hedgehog pathway activation might mediate pemetrexed resistance in NSCLC cells. Anticancer Res 40: 1451-1458, 2020.

28. Yang C, Zheng X, Ye K, Sun Y, Lu Y, Fan Q and Ge H: miR-135a inhibits the invasion and migration of esophageal cancer stem cells through the Hedgehog signaling pathway by targeting Smo. Mol Ther Nucleic Acids 19: 841-852, 2020.

29. Ma Y, Li G, Hu J, Liu X and Shi B: MicroRNA-494 regulates Gli3 expression and inhibits pancreatic cancer cells growth and migration. J Cell Biochem 119: 5324-5331, 2018.

30. Hyun J, Wang S, Kim J, Rao KM, Park SY, Chung I, Ha CS, Kim SW, Yun YH and Jung Y: MicroRNA-378 limits activation of hepatic stellate cells and liver fibrosis by suppressing Gli3 expression. Nat Commun 7: 10993, 2016. 
31. Liang Z, Liu Z, Cheng C, Wang H, Deng X, Liu J, Liu C, Li Y and Fang W: VPS33B interacts with NESG1 to modulate EGFR/PI3K/AKT/c-Myc/P53/miR-133a-3p signaling and induce 5-fluorouracil sensitivity in nasopharyngeal carcinoma. Cell Death Dis 10: 305, 2019.

32. Yang W, Ju HY and Tian XF: Circular RNA-ABCB10 suppresses hepatocellular carcinoma progression through upregulating NRP1/ABL2 via sponging miR-340-5p/miR-452-5p. Eur Rev Med Pharmacol Sci 24: 2347-2357, 2020

33. Shang H, Sun L, Braun T, Si Q and Tong J: Association between miR-124 rs531564 and miR-100 rs1834306 polymorphisms and cervical cancer: A meta-analysis. Eur J Gynaecol Oncol 40: 925-931, 2019.

34. Liu B, Shyr Y, Cai J and Liu Q: Interplay between miRNAs and host genes and their role in cancer. Brief Funct Genomics 18: 255-266, 2018.

35. Chen Y, Li C, Tan C and Liu X: Circular RNAs: A new frontier in the study of human diseases. J Med Genet 53: 359-365, 2016.
36. Zhi XH, Jiang K, Ma YY and Zhou LQ: OIP5-AS1 promotes the progression of gastric cancer cells via the miR-153-3p/ZBTB2 axis. Eur Rev Med Pharmacol Sci 24: 2428-2441, 2020.

37. Zuo J, Zhao M, Fan Z, Liu B, Wang Y, Li Y, Lv P, Xing L, Zhang $X$ and Shen H: MicroRNA-153-3p regulates cell proliferation and cisplatin resistance via Nrf-2 in esophageal squamous cell carcinoma. Thorac Cancer 11: 738-747, 2020.

38. Chang AC, Lien MY, Tsai MH, Hua CH and Tang CH: WISP-1 promotes epithelial-mesenchymal transition in oral squamous cell carcinoma cells via the $\mathrm{miR}-153-3 \mathrm{p} /$ Snail axis. Cancers (Basel) 11: 1903, 2019.

(i) (9) This work is licensed under a Creative Commons Attribution-NonCommercial-NoDerivatives 4.0 International (CC BY-NC-ND 4.0) License. 